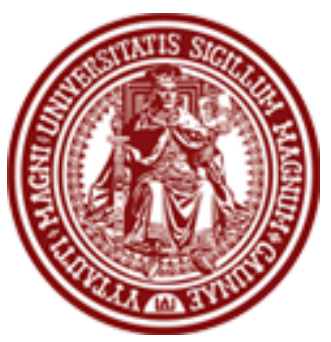

DE DE GRUYTER OPEN

\section{BALTIC JOURNAL OF LAW \& POLITICS}

A Journal of Vytautas Magnus University

VOLUME 10, NUMBER 1 (2017)

ISSN 2029-0454

\title{
THE DEFINITION OF BULLYING IN COMPULSORY EDUCATION: FROM A GENERAL TO A LEGAL PERSPECTIVE
}

\author{
Agnè Margevičiūtè \\ Lecturer, Ph.D. Candidate \\ Mykolas Romeris University, Faculty of Public Security (Lithuania) \\ Contact information \\ Address: V. Putvinskio str. 70, 44211 Kaunas, Lithuania \\ Phone: +37037303655 \\ E-mail address: agne4444@gmail.com
}

Received: December 4, 2016; reviews: 2; accepted: June 22, 2017.

\begin{abstract}
The definition of the word 'bullying' diverges based on the field of practice and research, in the absence of an agreed-upon overreaching definition. The latter would allow maximum flexibility in contributing to the variations in findings of various academic studies. Some argue that the lack of comprehensive definition is a factor of inaccuracy in estimating the prevalence of bullying itself. The 'definition' per se [of bullying] is in general recognized by the state law of the United States as one of the key components of any policy adopted by the states and local educational agencies, and which is required to be consistent with the definitions specified in state law. This article presents an overview of the definitions of bullying beyond the legal sphere in general as well as from a legal perspective. Special focus is dedicated to the state laws of the US as the main national jurisdiction that has adopted education law that contains explicit definition of bullying, as well as some of the aspects of defining bullying within the general and legal context of Lithuanian jurisdiction.
\end{abstract}




\section{KEYWORDS}

Right to education, bullying, definition of bullying, legal definition of bullying 


\section{INTRODUCTION}

Consistent interdisciplinary research efforts have led to breakthrough observations and conclusions in regard to bullying of learners at schools as a worldwide problem, ${ }^{1}$ addressed by educators, parents and legislators ${ }^{2}$ on national, regional and international levels. ${ }^{3}$ However, relevant and applicable to different aspects of education as a process in a young person's life, the extralegal approach of research in regard to bullying at school is prevailing, and the legal approach is insufficient. The same observation can be made in regard to the definition of bullying. Many researchers provide generalized extra legal definitions of the phenomenon, but there are very few jurisdictions where legislators are keen on adopting legal definitions of the term bullying.

The term "bullying" is without question a universally accepted term linguistically of what has been generally perceived as intentional aggressive behavior, mobbing, harassment, pestering, silent treatment, ignoring, etc. with an outcome of causing pain or discomfort of some extent. Scientific literature provides numerous concepts of the phenomenon of bullying. ${ }^{4}$ It is referred to as direct or indirect ${ }^{5}$ dehumanizing ${ }^{6}$ "delinquent behavior", 7 intentional harmful behavior, carried out repeatedly, against an individual who is unable to defend themselves, ${ }^{8}$ social interaction, ${ }^{9}$ physical or psychological abuse, ${ }^{10}$ to name a few. Yet a universal definition of bullying does not exist. However, the definition of bullying provided by Dan Olweus (who is perhaps the most prominent researcher of the ongoing global interdisciplinary effort in the field of school bullying) resonates in the vast majority of scholarly research on bullying.

There are few national jurisdictions that have passed relevant laws that deal with specific forms of bullying and the number of laws addressing the issue of

\footnotetext{
${ }^{1}$ Neville Harris, "Pupil Bullying, Mental Health and the Law in England": 31; in: Neville Harris and Paul Meredith, eds., Children, Education and Health, International Perspectives on Law and Policy (ASHGATE, 2005).

2 Kathryn S. Whitted and David R. Dupper, "Best Practices for Preventing or Reducing Bullying in Schools," Children \& Schools 27 (3) (2005): 167.

3 Aisling Parkes, Children and International Human Rights Law, The Right of the Child to be Heard (Routledge, 2013), 256.

${ }_{4}$ Saulius Girdvainis, "Patyčios tarp mokiniu bendrojo lavinimo mokykloje: samprata, dalyviai, priežastys ir padariniai" // http://www.su.It/bylos/mokslo_leidiniai/jmd/2013_2_40/girdvainis.pdf.

5 George M. Batche and Howard M. Knoff, "Bullies and Their Victims: Understanding A Pervasive Problem in the Schools," School Psychology Review 23 (2004): 167.

${ }^{6}$ Florida Senate Bill No. 114, Florida Statutes Annotated $\$ 1006.147$ (2007).

7 Vilija Targamadzè and Džiuginta Valeckienè, "Patyčiu bendrojo lavinimo mokykloje samprata: priežasčiu, formu ir pasekmiu diskursas" (Conception of Bullying at Comprehensive School: Discourse on Reasons, Forms and Consequences), Acta Paedagogica Vilnensia 19 (2007): 169.

8 Dan Olweus, Bullying at School: What We Know and What Can We Do (Oxford, England: Blackwell Publishers, 1993).

9 Stan Davis and Julia Davis, Schools Where Everyone Belongs: Practical Strategies for Reducing Bullying, $2^{\text {nd }}$ ed. (Champaign, IL: Research Press, 2007), 9.

10 Kathryn S. Whitted and David R. Dupper, supra note 2: 169.
} 
bullying directly is not abundant, but nevertheless increasing. One of the most advanced national jurisdictions where specific legislation explicitly addressing bullying has been adopted is the USA, where laws and policies governing bullying vary by state and entity. ${ }^{11}$ The article focuses on analysis of the state laws in education that deal with school violence issues and, thus, contain explicit definitions of bullying.

The aim of the article is to:

- analyze state laws on education of the US in regard to the legal definition of bullying;

- present the analysis of the correlation between the general definition of bullying provided by the scholars of extralegal fields of research and the legal definition of bullying provided by the state laws on education of the US;

Methods of systemic and analytical-critical, comparative analysis were applied for the research of the article. In addition, methods of documentary analysis and generalization were used.

\section{DEFINING BULLYING}

\subsection{BEYOND THE LEGAL SPHERE}

According to Olweus "a person is being bullied (or victimized) when he or she is exposed, repeatedly and over time, to negative actions on the part of one or more other persons."12 The ground rule definition provided by Olweus sets a clear component framework for an action to be considered bullying and has been applied and adhered to by researchers ever since. It is provided that action must be: 1 ) negative, with intention to inflict physical injury or discomfort by aggressive behavior; 2) repetitive and over time; 3 ) represent an imbalance of strength or power (physical and/or psychological). ${ }^{13}$ The component parts of the definition suggested by Dan Olweus are resonated by numerous researchers and practitioners. ${ }^{14}$

The definition of the concept of bullying per se should be recognized as another strong argument in favor of the newness of the relevance of the issue of

\footnotetext{
11 USLegal, Inc., "Bullying Law \& Legal Definition" (2001-2016) // http://definitions.uslegal.com/b/bullying/.

12 Dan Olweus, "Bullying - Victim Problems Among School Children: Basic Facts and Effects of a School Based Intervention Programs": 412; in: Debra J. Pepler and Kenneth H. Rubin, eds., Development and Treatment of Childhood Aggression (Hillsdale, NJ: Lawrence Elbraum Associates, Inc., 1991).

13 Dan Olweus, supra note 8, 8-9.

${ }^{14}$ Kathryn S. Whitted and David R. Dupper, supra note 2: 168; George M. Batche and Howard M. Knoff, supra note 5: 167; John H. Hoover, Ronald L. Oliver, and Keith A. Thomson, "Perceived victimization by school bullies: New research and future directions," Journal of Humanistic Education and Development 32 (1993): 76; Vilija Targamadzè and Džiuginta Valeckienè, supra note 7: 159.
} 
bullying in general. For instance, in the case of Lithuania, there is no precise linguistic term in the Lithuanian language that would describe the phenomenon of bullying ${ }^{15}$ or convey the various behavioral aspects in relation to the phenomenon. ${ }^{16}$ Thus, there still exists a problem of comprehensive definition of bullying. ${ }^{17}$ There are different terms that are used to identify the acts of bullying, such as, harass, insult, molest, harm, humiliate, tease, etc., in fact terms bullying and harassment are used as synonyms by some of the experts. ${ }^{18}$ Theoretically, bullying at school is also referred to as a psychological and pedagogical problem connected with public health, as well as children and upbringing, ${ }^{19}$ preconditioned by the low emotional literacy of the society. ${ }^{20}$ The extent of bullying is recognized as direct indicator of the mental health of the Lithuanian society. ${ }^{21}$ In 2007 a comprehensive definition of bullying, based on the theoretical approach of Dan Olweus, ${ }^{22}$ in Lithuanian was introduced by the experts of the field of educology and referred to it as "a conscious, unprovoked, continuous verbal or physical insult by one or several persons with intention to create permanent models of abuse and insults." 23

Another relevant observation definition-wise is imperative. The phenomenon of bullying at school prompted emergence of yet new term. Due to the effect that consequences of bullying have, the new term of 'bullycide' was introduced in 2001, 24 albeit still considered a relatively new term and gaining increasing interest. ${ }^{25}$ 'Bullycide' refers to an act of suicide committed by a student because he or she deemed it less painful than attending school and being bullied, taunted and

15 Albinas Bagdonas and Indré Padarauskaitè, "Paaugliu kūno vaizdas ir patyčiu patirtis" (Body-Image and Bullying in Adolescents), Psichologija 46 (2012): 45.

16 Živilè Vilma Jonynienè, Agnè Bartkutè, and Tomas Butvilas, "Teisinis švietimas kaip prevencinè priemonè prieš patyčias mokyklose: mokiniu ir mokytoju sampratos" (Legal Education as the Prevention for Bullying at Schools: Conceptions of Students and Teachers), Socialinis darbas/Social Work 10(2) (2011): 220.

17 Giedrius Girdvainis and Rasa Pocevičienè, "Patyčiu problema ir jos prevencijos bendrojo lavinimo mokykloje analizé" (Analysis of Problem of Bullying and its Prevention at Comprehensive School), Socialiniai mokslai. Jaunuju mokslininku darbai 4(25) (2009): 133.

18 Robertas Povilaitis and Jurgita Valiukevičiūtè, Patyčiu prevencija mokyklose (Prevention of Bullying at Schools), edited by Laima Bulotaitè (Vilnius: Multiplex, 2006), 6.

19 Apolinaras Zaborskis, Lina Cirtautienè, and Nida Žemaitienè, "Bullying in Lithuanian schools in 19942002," Medicina (Kaunas) 41(7) (2005): 614.

20 Dainius Pūras, "Foreword": 5; in: Robertas Povilaitis and Jurgita Valiukevičiūtè, Patyčiu prevencija mokyklose (Prevention of Bullying at Schools) (Vilnius: Multiplex, 2006).

${ }^{21}$ Apolinaras Zaborskis, et al. for WHO/HBSC Forum, "Lithuania: Youth Mental Health - From Research to Policies, Practice and Partnerships" (2007) //

http://www.euro.who.int/_data/assets/pdf_file/0006/74769/Hbsc_Forum_2007_Lithuania.pdf.

22 Dan Olweus, "Bully/Victim Problems in School: Facts and Intervention," European Journal of Psychology of Education Vol. XII (1997): 496-497.

${ }^{23}$ Vilija Targamadzè and Džiuginta Valeckienè, supra note 7: 159.

${ }^{24}$ Neil Marr and Tim Field, Bullycide: Death at Playtime - An Expose' of Child Suicide Caused by Bullying (Success Unlimited, 2001), 9-12.

25 Pamela Kulbarsh, "Bullycide: Suicide as a Result of Bullying" (January 2012) //

http://www.officer.com/article/10611621/bullycide-suicide-as-a-result-of-bullying. 
humiliated. ${ }^{26}$ The latter term represents the most severe impact of bullying on the victims - ending life.

It can be concluded that bullying is in general defined more by its character, adhering to the framework provided by Dan Olweus, than by a concrete linguistic meaning. For example, any action that can be described by a concrete linguistic term (harassment, teasing, name calling, etc.) will be considered bullying if the action has the characteristics of bullying. The term may also be too narrow, because the definition does not fully disclose what it actually is in regard to its form. For example, a student is harassed by several classmates by ignoring that student, and in this case it will be difficult for that student to defend his or her case of being bullied against, because of the underlying character of the actions of the classmates. Therefore, researchers still recognize the problem of defining the phenomenon of bullying because of many linguistic terms that are actually related to what bullying is. ${ }^{27}$ Additionally, the cross cultural aspect of how bullying is perceived, may hinder a uniform conceptualization of bullying in transnational studies of bullying. ${ }^{28}$ Thus, the multidimensional character of the term of bullying, according to research, only confirms the problem of naming the complexity of the phenomenon, also resulting in the fact that there is no uniform classification of the forms of bullying. ${ }^{29}$ However, some researchers approve the absence of the overreaching definition in the interests of flexibility ${ }^{30}$ and efficiency of school efforts in adopting and applying preventive measures locally. ${ }^{31}$

\subsection{FROM A LEGAL PERSPECTIVE}

The term bullying, though not defined, first appeared in education law of England in the School Standards and Framework Act of 1998 in the context of prescribing responsibilities to the governing bodies and head teachers of schools in preventing all forms of bullying among pupils. ${ }^{32}$ Bullying is also explicitly cited in subsequent education law acts of 2006 and $2010^{33}$ in the context of imperative

\footnotetext{
26 Paul Timm, School Security, How to Build and Strengthen a School Safety Program, edited by Brian Romer (Elsevier, 2015), 108.

27 Vilija Targamadzè and Džiuginta Valeckienè, supra note 7: 164.

28 Kayleigh L. Chester, Mary Callaghan, Alina Cosma, Peter Donnelly, Wendy Craig, Sophie Walsh, and Michal Molcho, "Cross-national time trends in bullying victimization in 33 countries among children aged 11, 13 and 15 from 2002 to 2010," European Journal of Public Health Vol. 25 (Supplement 2, 2015): 61 // DOI: doi:10.1093/eurpub/ckv029.

${ }^{29}$ Vilija Targamadzè and Džiuginta Valeckienè, supra note 7: 164.

30 Neville Harris, supra note 1: 34.

31 Chris Watkins and Patsy Wagner, Improving School Behavior (London: Paul Chapman, 2000), 49.

32 The Stationery Office Limited, "School Standards and Framework Act 1998" (July 1998) // http://www.legislation.gov.uk/ukpga/1998/31/pdfs/ukpga_19980031_en.pdf.

33 Education and Inspections Act 2006, The Education (Independent School Standards) Regulations of 2010 and 2014.
} 
obligation of state schools to have measures of bullying prevention ${ }^{34}$ and requirement to ensure adoption and implementation of anti-bullying strategies by private schools. ${ }^{35}$

Another jurisdiction where bullying has been explicitly addressed in state legislation for over a decade is that of the United States, ${ }^{36}$ where all states have passed state education laws tackling the issue of bullying at school, while a majority of these states have passed both relevant laws and policies. ${ }^{37}$ According to the components of the state anti-bullying determined by the interagency of the US Department of Education, 'specification of prohibited conduct' had been identified as one of the components. ${ }^{38}$ The US states' legislature is by far the most extensive resource of legal acts in education law, explicitly addressing bullying at school. The latter circumstance allows for an in-depth analysis of how the legal definition of bullying correlates with the universally accepted and applied definition of bullying (by Olweus) and its component parts.

\section{THE LEGAL DEFINITION OF BULLYING IN THE STATE LEGISLATION} OF THE UNITED STATES

A thorough analysis of all states' laws on education that address violence at schools and that contain explicit or indirect definitions of bullying has been carried out. Only those provisions of the state law on education that contained the actual definition of bullying were analyzed in regard to the three generally accepted definitional components of bullying.

Table 1: Analysis of the legal definitions of bullying, as defined by the state law of the US, in relation with the universally and generally applied definition of bullying, according to Dan Olweus

\begin{tabular}{|c|c|c|}
\hline $\begin{array}{c}\text { The three component parts universally and generally applied to the definition of } \\
\text { bullying, according to Dan Olweus, in regard to explicit definitions of the term } \\
\text { bullying }\end{array}$ \\
\hline $\begin{array}{c}\text { Negative, with intention to inflict } \\
\text { physical injury or discomfort by } \\
\text { aggressive behavior }\end{array}$ & $\begin{array}{r}\text { Repetitive and over } \\
\text { time }\end{array}$ & $\begin{array}{c}\text { Represent an } \\
\text { imbalance of strength } \\
\text { (physical and/or } \\
\text { psychological) }\end{array}$ \\
\hline
\end{tabular}

${ }^{34}$ Education and Inspections Act (2006) //

http://www.legislation.gov.uk/ukpga/2006/40/pdfs/ukpga_20060040_en.pdf.

35 The Education (Independent School Standards) Regulations (2010) // http://www.legislation.gov.uk/uksi/2010/1997/pdfs/uksi_20101997_en.pdf.

36 By 201045 states have passed specific bullying prevention legislation. Source: US Department of Education, "Key Policy Letters from the Education Secretary and Deputy Secretary" (December 2010) // http://www2.ed.gov/policy/gen/guid/secletter/101215.html.

37 "Policies \& Laws," stopbullying.gov (May 2015) // http://www.stopbullying.gov/laws/index.html.

38 "Key Components in State Anti-Bullying Laws," stopbullying.gov (March 2014) // http://www.stopbullying.gov/laws/key-components/index.html. 


\begin{tabular}{|c|c|c|}
\hline $\begin{array}{l}\text { 'Intentional behavior,' which 'places the } \\
\text { victim in 'reasonable fear of harm.'39 }\end{array}$ & 'Continuous pattern.' & - \\
\hline $\begin{array}{l}\text { 'Intentional written, oral, or physical act } \\
{[\ldots] \text { with intent of threatening, }} \\
\text { intimidating, harassing, or frightening', } \\
\text { and which 'physically harms. }{ }^{40}\end{array}$ & - & - \\
\hline $\begin{array}{l}\text { 'Intentional harassment, intimidation, } \\
\text { humiliation, ridicule, defamation, or } \\
\text { threat, or incitement of violence.' } 41\end{array}$ & - & - \\
\hline $\begin{array}{l}\text { 'Severe or pervasive physical or verbal } \\
\text { act or conduct,' causing 'substantially } \\
\text { detrimental effect on }[\ldots] \text { physical or } \\
\text { mental health.' } 42\end{array}$ & $\begin{array}{l}\text { 'One or more acts by } \\
\text { a pupil or group of } \\
\text { pupils.' }\end{array}$ & - \\
\hline $\begin{array}{l}\text { 'Written or verbal expression, or physical } \\
\text { or electronic act or gesture, or a pattern } \\
\text { thereof, that is intended to coerce, } \\
\text { intimidate, or cause any physical, } \\
\text { mental, or emotional harm to any } \\
\text { student.' } 43\end{array}$ & $\begin{array}{l}\text { A notion of } \\
\text { 'reasonable balance } \\
\text { between the pattern } \\
\text { and severity of the } \\
\text { bullying behavior' is } \\
\text { suggested for } \\
\text { inclusion into the } \\
\text { policy provisions. }\end{array}$ & - \\
\hline $\begin{array}{l}\text { 'written, oral or electronic } \\
\text { communication,' 'physical act or gesture } \\
\text { by one or more students,' 'causes } \\
\text { physical or emotional harm, }{ }^{\prime 44}\end{array}$ & $\begin{array}{l}\text { Refers to 'the } \\
\text { repeated use by one } \\
\text { or more students,' } \\
\text { 'repeatedly directed } \\
\text { at another student.' }\end{array}$ & - \\
\hline $\begin{array}{l}\text { 'Intentional written, electronic, verbal or } \\
\text { physical act or actions' that cause } \\
\text { 'reasonable fear of substantial harm.'45 }\end{array}$ & - & - \\
\hline $\begin{array}{l}\text { Infliction of 'physical hurt or } \\
\text { psychological distress.' 'Teasing, social } \\
\text { exclusion, threat, intimidation, stalking, } \\
\text { physical violence, theft, sexual, religious, } \\
\text { or racial harassment, public or private }\end{array}$ & $\begin{array}{l}\text { Is carried out } \\
\text { 'systematically and } \\
\text { chronically.' }\end{array}$ & - \\
\hline
\end{tabular}

39 Alabama House Bill No. 216, The Alabama Student Harassment Prevention Act No. 2009-571, Alabama Code §16-28B-3 (2009).

40 Alaska House Bill No.482, Harassment, Intimidation, and Bullying Policy, Alaska Statute §14.33.250 (2006).

41 Arkansas House Bill No. 1708, Act to Define Bullying and Cyberbullying, Arkansas Code Annotated §618-514 (2005).

42 California Assembly Bill No. 606, California Education Code $§ 48900.1$ (2008).

43 Colorado Senate Bill No. 01-080, Concerning the Prevention of Bullying, Colorado Revised Statutes $\S 22-32-109.1$ (1) (b) (2001).

${ }_{44}$ Connecticut House Bill No. 5563, Connecticut General Statutes §10-222d (2006).

45 Delaware House Bill No. 7, Delaware Code Annotated Title 14 §4112D (2007). 


\begin{tabular}{|c|c|c|}
\hline $\begin{array}{l}\text { humiliation, destruction of property' are } \\
\text { specifically referred to. }{ }^{46}\end{array}$ & & \\
\hline $\begin{array}{l}\text { 'Willful attempt or threat to inflict injury,' } \\
\text { 'intentional written, verbal, or physical } \\
\text { act' that causes 'substantial physical } \\
\text { harm.' }{ }^{\prime 47}\end{array}$ & - & $\begin{array}{l}\text { 'Accompanied by an } \\
\text { apparent present } \\
\text { ability to do so.' }\end{array}$ \\
\hline $\begin{array}{l}\text { 'Any written, verbal, graphic, or physical } \\
\text { act,' that 'causes mental or physical } \\
\text { harm' and is 'sufficiently severe }[\ldots] \text { or } \\
\text { pervasive. }{ }^{\prime 48}\end{array}$ & $\begin{array}{l}\text { 'Sufficiently } \\
\text { persistent.' }\end{array}$ & - \\
\hline $\begin{array}{l}\text { 'Any intentional gesture, or any } \\
\text { intentional written, verbal or physical act } \\
\text { or threat by a student,' that is } \\
\text { 'sufficiently severe, persistent or } \\
\text { pervasive' and has effect of 'harming a } \\
\text { student' or 'damaging student's } \\
\text { property. }{ }^{49}\end{array}$ & $\begin{array}{l}\text { 'Sufficiently } \quad[\ldots] \\
\text { persistent.' }\end{array}$ & - \\
\hline $\begin{array}{l}\text { 'Any severe or pervasive physical or } \\
\text { verbal act or conduct' that causes } \\
\text { 'reasonable fear of harm to the student's } \\
\text { or students' person or property,' as well } \\
\text { as 'substantially detrimental effect on } \\
\text { student's or students' physical or mental } \\
\text { health. } 50\end{array}$ & - & - \\
\hline $\begin{array}{l}\text { 'Overt, unwanted }[\ldots] \text { acts or gestures,' } \\
\text { 'physical acts }[\ldots] \text {, aggression, or any } \\
\text { other behaviors, }[\ldots] \text { with the intent to } \\
\text { harass, ridicule, humiliate, intimidate, or } \\
\text { harm. }{ }^{51}\end{array}$ & 'Repeated acts.' & - \\
\hline $\begin{array}{l}\text { 'Any electronic, written, verbal, or } \\
\text { physical act or conduct [...] and which } \\
\text { creates an objectively hostile school } \\
\text { environment,' causes 'reasonable fear of } \\
\text { harm to the student's or students' } \\
\text { person or property,' as well as } \\
\text { 'substantially detrimental effect on }\end{array}$ & - & - \\
\hline
\end{tabular}

46 Florida Senate Bill No. 114, Florida Statutes Annotated $\S 1006.147$ (2007).

47 Georgia House Bill No. 84, Georgia Code Annotated §20-2-751.4 (1999).

48 Hawaii Administrative Rules, Department of Education §8-19-2.

49 Idaho House Bill No. 750aa, Jared's Law, Idaho Code \$18-917a (2006).

50 Illinois Senate Bill No. 1026, 105 Illinois Compiled Statutes §5/27-23.7 (2006).

51 Indiana Senate Enrolled Act, Indiana Code Annotated §20-33-8-0.2 (2005). 


\begin{tabular}{|c|c|c|}
\hline $\begin{array}{l}\text { student's or students' physical or mental } \\
\text { health. }{ }^{52}\end{array}$ & & \\
\hline $\begin{array}{l}\text { 'Intentional gesture or any intentional } \\
\text { written, verbal, electronic or physical } \\
\text { act,' 'sufficiently severe [...] or pervasive } \\
\text { 'harming [...] physically or mentally,' } \\
\text { 'damaging property.'53 }\end{array}$ & $\begin{array}{l}\text { 'Sufficiently } \\
\text { persistent.' }\end{array}$ & - \\
\hline $\begin{array}{l}\text { 'Any unwanted verbal, physical, or social } \\
\text { behavior.'54 }\end{array}$ & $\begin{array}{l}\text { 'Is repeated or has } \\
\text { the potential to be } \\
\text { repeated.' }\end{array}$ & $\begin{array}{l}\text { 'Involves a real or } \\
\text { perceived } \\
\text { imbalance.' }\end{array}$ \\
\hline $\begin{array}{l}\text { 'Gestures, including but not limited to } \\
\text { obscene gestures and making faces,' } \\
\text { 'including but not limited to calling } \\
\text { names, threatening harm, taunting, } \\
\text { malicious teasing, or spreading untrue } \\
\text { rumors,' 'physical acts.'55 }\end{array}$ & 'A pattern.' & - \\
\hline $\begin{array}{l}\text { 'written, oral or electronic expression or } \\
\text { a physical act or gesture,' 'physically } \\
\text { harming,' 'placing a student in } \\
\text { reasonable fear of physical harm or } \\
\text { damage to the student's property. } 56\end{array}$ & - & - \\
\hline $\begin{array}{l}\text { 'Intentional written, verbal, or physical } \\
\text { act,' 'physically harms,' 'damages [...] } \\
\text { property.'57 }\end{array}$ & - & - \\
\hline $\begin{array}{l}\text { 'Written, verbal or electronic expression } \\
\text { or a physical act or gesture or any } \\
\text { combination thereof,' 'causes physical or } \\
\text { emotional harm to the victim,' 'damage } \\
\text { to the victim's property,' 'places the } \\
\text { victim in reasonable fear of harm.'58 }\end{array}$ & 'Repeated use.' & - \\
\hline $\begin{array}{l}\text { 'Any written, verbal, or physical act,' } \\
\text { 'having an actual and substantial } \\
\text { detrimental effect on a pupil's physical or } \\
\text { mental health.'59 }\end{array}$ & - & - \\
\hline
\end{tabular}

52 Iowa Senate File No. 61, Iowa Code $\$ 280.28$ (2007).

53 Kansas House Bill No. 2310, Kansas Statutes Annotated §72-8256 (2008).

54 Kentucky House Bill No. 91, Chapter No. 25, The Golden Rule Act, Kentucky Revised Statues Annotated $\S 158.148$ (2008).

55 Louisiana Laws Revised Statue, Louisiana Revised Statutes Annotated §17:416.13 (2010).

56 Maine Revised Statute Annotated, Title 20-A §6554 (2011).

57 Maryland House Bill No. 199, Maryland Education Code Annotated §7-424.3 (2008).

58 Senate Bill No. 2404, Chapter 92, Bullying in Schools Act, Massachusetts General Laws Chapter §71, Section 370 (2010).

59 Michigan House Bill No. 241, Michigan Compiled Laws §380.1310b (2011). 


\begin{tabular}{|c|c|c|}
\hline $\begin{array}{l}\text { 'Objectively offensive,' 'intimidating, } \\
\text { threatening, abusive, or harming } \\
\text { conduct. } 60\end{array}$ & - & $\begin{array}{l}\text { 'There is an actual or } \\
\text { perceived imbalance } \\
\text { of power between the } \\
\text { student engaging in } \\
\text { prohibited conduct } \\
\text { and the target of the } \\
\text { behavior.' }\end{array}$ \\
\hline $\begin{array}{l}\text { 'Gestures or written, electronic or verbal } \\
\text { communications, or any physical act or } \\
\text { any threatening communication,' that } \\
\text { 'places a student or school employee in } \\
\text { actual and reasonable fear of harm' to } \\
\text { self and property. } 61\end{array}$ & $\begin{array}{l}\text { 'Any pattern of } \\
\text { gestures.' }\end{array}$ & - \\
\hline $\begin{array}{l}\text { 'Intimidation or harassment that causes } \\
\text { a reasonable student to fear for his or } \\
\text { her physical safety or property. }{ }^{\prime 62}\end{array}$ & - & - \\
\hline $\begin{array}{l}\text { 'Any harassment, intimidation, hazing, or } \\
\text { threatening, insulting, or demeaning } \\
\text { gesture or physical contact,' 'causes } \\
\text { harm,' 'damages property.' } 63\end{array}$ & $\begin{array}{l}\text { 'Persistent, severe, } \\
\text { or repeated.' }\end{array}$ & - \\
\hline 'Physical, verbal, or electronic abuse.'64 & 'Ongoing pattern.' & - \\
\hline $\begin{array}{l}\text { 'Written, verbal or electronic expressions } \\
\text { or physical acts or gestures,' 'single } \\
\text { severe and willful act or expression,' } \\
\text { 'taunting, name-calling, belittling, } \\
\text { mocking or use of put-downs or } \\
\text { demeaning humor.'65 }\end{array}$ & $\begin{array}{l}\text { 'Repeated } \\
\text { pervasive...' }\end{array}$ & - \\
\hline $\begin{array}{l}\text { 'Written, verbal, or electronic } \\
\text { communication, or a physical act or } \\
\text { gesture,' which 'physically harms,' } \\
\text { 'damages,' 'causes emotional distress.'66 }\end{array}$ & $\begin{array}{l}\text { 'A single significant } \\
\text { incident or a pattern } \\
\text { of incidents.' }\end{array}$ & - \\
\hline $\begin{array}{l}\text { 'Any gesture, any written, verbal or } \\
\text { physical act, or any electronic } \\
\text { communication, whether it be a single } \\
\text { incident or a series of incidents }[. . .] \text { that } \\
\text { that substantially disrupts or interferes }\end{array}$ & - & - \\
\hline
\end{tabular}

60 Minnesota House File No. 826, Minnesota Statute §121A.031 (2014).

${ }^{61}$ Mississippi Senate Bill No. 2015, Bullying and Harassing Behavior in Public Schools, Mississippi Code Annotated §37-11-67 (2010).

62 Missouri Senate Bill No. 894, Missouri Revised Statutes $\$ 160.775$ (2006).

63 Montana Code Annotated, L. 2015, En. Sec. 2, Ch. 253, §20-5-208 (2015).

64 Nebraska Legislative Bill No. 205, Nebraska Revised Statutes $\$ 79-2,137$ (2008).

65 Nevada Assembly Bill, Nevada Revised Statutes Annotated §388.122 (2009).

66 New Hampshire Senate Bill No. 360, New Hampshire Revised Statutes Annotated §193-F:3 (2000). 


\begin{tabular}{|c|c|c|}
\hline $\begin{array}{l}\text { with the orderly operation of the school } \\
\text { or the rights of other students. }{ }^{67}\end{array}$ & & \\
\hline $\begin{array}{l}\text { 'Any [...] verbal or electronic expression, } \\
\text { physical act or gesture, or a pattern } \\
\text { thereof, that is intended to cause } \\
\text { distress. }{ }^{68}\end{array}$ & $\begin{array}{ll}\text { 'Repeated } & \text { and } \\
\text { pervasive } & {[\ldots]} \\
\text { pattern.' } & \end{array}$ & - \\
\hline $\begin{array}{l}\text { 'Gestures or written, electronic, or verbal } \\
\text { communications, or any physical act or } \\
\text { any threatening communication.'69 }\end{array}$ & 'Pattern.' & - \\
\hline $\begin{array}{l}\text { 'So severe, pervasive, or objectively } \\
\text { offensive that it substantially interferes } \\
\text { with the student's educational } \\
\text { opportunities,' 'places the student in } \\
\text { actual and reasonable fear of harm' to } \\
\text { self an property. }{ }^{70}\end{array}$ & - & - \\
\hline $\begin{array}{l}\text { 'Any intentional written, verbal, } \\
\text { electronic, or physical act that [...] } \\
\text { causes mental or physical harm to the } \\
\text { other student,' is 'sufficiently severe, or } \\
\text { pervasive. }{ }^{71}\end{array}$ & $\begin{array}{l}\text { 'Sufficiently } \quad[\ldots] \\
\text { persistent.' }\end{array}$ & - \\
\hline $\begin{array}{l}\text { 'Any }[\ldots] \text { harassment, intimidation, } \\
\text { threatening behavior, physical acts, } \\
\text { verbal or electronic communication }[. . .] \\
\text { that results in or is }[\ldots] \text { done with the } \\
\text { intent to cause negative educational or } \\
\text { physical results. }{ }^{\prime 72}\end{array}$ & 'Pattern.' & - \\
\hline $\begin{array}{l}\text { 'Any act that Substantially interferes } \\
\text { with a student's educational benefits, } \\
\text { opportunities or performance' and has } \\
\text { 'physically harming' effect. }{ }^{73}\end{array}$ & - & - \\
\hline $\begin{array}{l}\text { 'An intentional electronic, written, verbal } \\
\text { or physical act, or a series of acts,' } \\
\text { 'severe [...] or pervasive,' 'substantially } \\
\text { interfering with a student's education. }{ }^{74}\end{array}$ & 'Persistent.' & - \\
\hline
\end{tabular}

67 New Jersey Assembly Bill No. 3466, Chapter No. 122, N.J. Stat. Ann. §18A-37-14 (2011).

68 New Mexico Department of Public Education Rule Title 6, Chap. 12, Part 7, Bullying Prevention, New Mexico Administrative Code $\S 6.12 .7 .7$ (2006).

69 North Carolina Senate Bill No. 526, School Violence Prevention Act, N.C. Gen. Stat. §115C-407.5 (2006).

70 North Dakota House Bill No. 1465, North Dakota Century Code Chapter §15.1-19-17 (2011).

71 Ohio House Bill No. 276, Harassment, Intimidation, and Bullying Policy, Adopt Own, Ohio Rev. Stat. Ann. $\S 3313.666$ (2006).

72 Oklahoma House Bill No. 2215, School Bullying Prevention Act, Okla. Stat. Ann. Tit. 70, §24-100.3 (2002).

73 Oregon House Bill No. 2599, Or. Rev. Stat. §339.351 (2009).

74 Pennsylvania House Bill No. 1067, 24 Pennsylvania Consolidated Statute §13-1303.1-A (2008). 


\begin{tabular}{|c|c|c|}
\hline $\begin{array}{l}\text { 'A gesture, an electronic communication, } \\
\text { or a written, verbal, physical, or sexual } \\
\text { act,' 'harming a student physically or } \\
\text { emotionally or damaging a student's } \\
\text { property. }{ }^{75}\end{array}$ & - & - \\
\hline $\begin{array}{l}\text { 'Causes physical hurt or psychological } \\
\text { distress.'76 }\end{array}$ & $\begin{array}{l}\text { 'Pattern of repeated } \\
\text { conduct.' }\end{array}$ & - \\
\hline $\begin{array}{l}\text { 'Any act that substantially interferes with } \\
\text { a student's educational benefits, } \\
\text { opportunities or performance.'77 }\end{array}$ & - & - \\
\hline 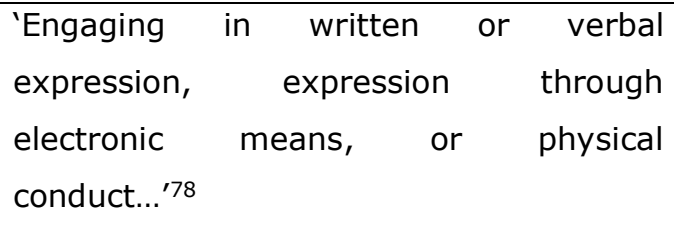 & & $\begin{array}{l}\text { 'Exploits an imbalance } \\
\text { of power between the } \\
\text { student perpetrator } \\
\text { and the student } \\
\text { victim.' }\end{array}$ \\
\hline $\begin{array}{l}\text { 'Intentionally or knowingly committing } \\
\text { an act that }[\ldots] \text { endangers the physical } \\
\text { health or safety of a school employee or } \\
\text { student. }^{79}\end{array}$ & - & - \\
\hline $\begin{array}{l}\text { 'Any overt act or combination of acts, } \\
\text { including an act conducted by electronic } \\
\text { means' and 'is intended to ridicule, } \\
\text { humiliate, or intimidate the student. }{ }^{80}\end{array}$ & $\begin{array}{l}\text { 'Is repeated over } \\
\text { time.' }\end{array}$ & - \\
\hline $\begin{array}{l}\text { 'Any aggressive and unwanted behavior } \\
\text { that is intended to harm, intimidate, or } \\
\text { humiliate the victim }[\ldots] \text { or causes } \\
\text { severe emotional trauma. }{ }^{81}\end{array}$ & $\begin{array}{l}\text { 'Is repeated over } \\
\text { time.' }\end{array}$ & $\begin{array}{l}\text { 'Involves a real or } \\
\text { perceived power } \\
\text { imbalance between } \\
\text { the aggressor or } \\
\text { aggressors and } \\
\text { victim.' }\end{array}$ \\
\hline $\begin{array}{l}\text { 'Any intentional electronic, written, } \\
\text { verbal, or physical act,' 'so severe, [...] } \\
\text { pervasive.' } 82\end{array}$ & 'So [...] persistent.' & - \\
\hline $\begin{array}{l}\text { 'Any intentional gesture, or any } \\
\text { intentional electronic, written, verbal or } \\
\text { physical act, communication, }\end{array}$ & $\begin{array}{l}\text { 'Sufficiently } \\
\text { persistent.' }\end{array}$ & - \\
\hline
\end{tabular}

75 South Carolina House Bill No. 3573, Safe School Climate Act, South Carolina Code Annotated §59-63120 (2006).

76 South Dakota Codified Law, §13-32-15 (2012).

77 Tennessee Senate Bill No. 283, Tenn. Code Ann. §49-6-4502 (2009).

78 Texas House Bill No. 1942, Texas Education Code Annotated $\S 37.0832$ (2011).

79 Utah House Bill No. 325, Utah Code Annotated §53A-11a-102 (2008)

80 Vermont House Bill No. H629, Vermont Statute Annotated Title 16, §11(a)(32) (2004).

81 Virginia Code Annotated, § 22.1-276.01 (2013).

82 Washington Substitute House Bill No. 2801, Harassment, Intimidation, and Bullying, Washington Revised Code §28A.300.285 (2010). 


\begin{tabular}{|l|l|l|}
\hline $\begin{array}{l}\text { transmission or threat,' 'sufficiently } \\
\text { severe }[\ldots] \text { or pervasive.'83 }\end{array}$ & & \\
\hline 'Any intentional gesture, any intentional & 'Sufficiently & - \\
electronic communication or any & persistent.' \\
intentional written, verbal or physical & & \\
act,' 'harming a student physically or & & \\
emotionally, damaging [...] property,' & & \\
'insulting or demeaning,' 'sufficiently & & \\
severe $[\ldots]$ or pervasive.'84 & & \\
\hline
\end{tabular}

Analysis of the states' law on education allows for the following observations in regard to how the legal definition of bullying corresponds to the generally accepted definition of bullying used by scholars of the extralegal fields of research.

\subsection{ON NEGATIVITY}

Dan Olweus explains the notion of 'negative actions' as an attempt or an action committed with intent to cause injury or discomfort to the victim. ${ }^{85}$ The assessment of the legal definitions of bullying embedded in the laws of states of the US compared to the generally accepted definition of bullying (which provides a certain component framework) allows for the following conclusions, especially in regard to the aspects of repetitiveness of the act of bullying and imbalance of (physical or psychological) power between the victim and the bully. It is apparent that all the legal definitions by the state law correspond to the negative impact of the phenomenon of bullying in the school environment. It is perceived as 'intentional,' 'aggressive,' 'demeaning,' 'dehumanizing,' 'intimidating,' 'unwanted,' etc. However, as can be seen from Table 1, not all states' legislations equally refer to the repeated incidence of bullying, as well as presence of imbalance of physical or psychological power between the perpetrator and the victim in the definitions of bullying or other notions related to it.

\subsection{ON INCIDENCE}

Laws of fourteen states explicitly refer to the notions of continuity, repetitiveness, pattern, chronic behavior, extended periods of time, and that these elements be included in the definition of bullying. State law in instances where the definition of bullying does not explicitly require that it be subjected to a repeated

\footnotetext{
83 West Virginia House Bill No. 3225, West Virginia Code Annotated §18-2C-2 (2011).

84 Wyoming House Bill No. 0223, Wyo. Stat. Ann. §21-4-312 (2009).

85 Dan Olweus, supra note 8, 11.
} 
act or behavior over time does provide for certain characteristic of the said behavior in order for it to be considered as bullying. For example, the laws of nine states explicitly require for the act to be 'sufficiently persistent.' The latter notion can be interpreted with an inclination for an act of bullying to be of repetitive character, since the definition of the adjective persistent refers to an occurrence that is "continuing to exist or endure over a prolonged period." ${ }^{86}$ Yet, as per definition, the same persistent character can be applied to a single course of action in spite difficulty or opposition. ${ }^{87}$ Therefore, the persistent character can be applicable in both instances of multiple or single incidence of an act of bullying.

Thus, the legislation at large deviates from one of the component parts (i.e., repeated incidence over time) of the universally applied definition and does not explicitly require for an act to be repetitive and over time in order to be perceived as bullying. It is to be noted, however, that in all other instances where the state law definition of bullying at school does not make any reference to repetitiveness or duration of bullying, it is provided that the act (or acts) be sufficiently 'significant,' 'severe,' or 'pervasive,' or a 'single incident' or 'act' be 'significant.' In some instances state law places a strict obligation to include a reasonable balance between the pattern and the severity' of bullying behavior in the district's school policy. ${ }^{88}$

\subsection{ON IMBALANCE OF POWER}

Only five states included an explicit provision in regard to the presence of real or perceived power disparities between the aggressor and the victim of bullying. This resembles some of the emerging tendencies, based on the research of the acts of bullying at school, which provide that although a power imbalance usually increases the severity of the impact on the victim, a lack of power imbalance does not shield victims from the negative effects of bullying. ${ }^{89}$ Thus, the legal definition in the instance of the imbalance of power also deviates from the generally applied definition of bullying provided by the experts.

\footnotetext{
86 Oxford University Press, "Definition of persistent in English" (2016) //

http://www.oxforddictionaries.com/us/definition/american_english/persistent.

87 Ibid.: 203.

${ }^{88}$ Colorado Senate Bill, supra note 43.

${ }^{89}$ Heather A. Turner, David Finkelhor, Anne Shattuck, Sherry Hamby, and Kimberly Mitchell, "Beyond Bullying: Aggravating Elements of Peer Victimization Episodes," School Psychology Quarterly Vol. 30(3) (September 2015) // http://dx.doi.org/10.1037/spq0000058.
} 


\subsection{ON OTHER ASPECTS OF THE LEGAL DEFINITIONS OF BULLYING IN THE STATE LAWS OF THE US}

\subsubsection{VICTIMIZATION}

Some definitions include specifications of the aggressor. For example, Massachusetts state law defines bullying as repeated written, verbal or electronic expression or a physical act or gesture "by one or more students or by a member of a school staff," 90 while Vermont legislature defines bullying as an act "directed against a student by a student." 91 This shows possible differences in how the legislator perceives the aggressor of an act of bullying. Clear perception of the aggressor is important, because the state law provisions carry supremacy over the provisions of anti-bullying policies of the states' and schools. If the aggressor is defined solely as a student by law, it means that such perception shall carry over to subsequent state anti-bullying policies, internal school regulations, and a notion that anyone, besides a student, may be at fault of committing an act of bullying at school against a student will be 'lost in translation.'

The same remarks are relevant in regard to the perception of the victim of bullying at school. For example, very few state jurisdictions define bullying as an act or actions against "another student, school volunteer or school employee" (Delaware, ${ }^{92}$ Florida ${ }^{93}$ ), or "any/another person" (Georgia, ${ }^{94}$ Kentucky ${ }^{95}$ ), "any student or a staff member" (Iowa ${ }^{96}$ ), or as an act that "interferes with [...] the rights of other students" (New Jersey ${ }^{97}$ ), or "physical harm to a public school employee or student" (Arkansas ${ }^{98}$ ). Reportedly, as many as $25 \%$ of school staff members experience bullying, ${ }^{99}$ while $5-9 \%$ of teachers indicate that they were threatened with injury or physically attacked by students. ${ }^{100}$ However, the vast majority of the states' legislation explicitly refers to the victim of bullying as one student. This poses an oversight in regard to the safety of teachers (as well as other members of the school community), because focusing solely on student victimization and excluding teacher (or other school staff member) victimization

\footnotetext{
90 The definition of bullying also elaborates on school staff, stating that the latter is includes, but is not limited to, an educator, administrator, school nurse, cafeteria worker, custodian, bus driver, athletic coach, advisor to an extracurricular activity or paraprofessional.

91 Vermont House Bill, supra note 80.

92 Delaware House Bill, supra note 45

93 Florida Senate Bill, supra note 46.

94 Georgia House Bill, supra note 47.

95 Kentucky House Bill, supra note 54.

96 Iowa Senate File, supra note 52.

97 New Jersey Assembly Bill, supra note 67.

98 Arkansas House Bill, supra note 41.

99 Cindy Long, "Bulliyng of Teachers Pervasive in Many Schools" (May 2012) // http://neatoday.org/2012/05/16/bullying-of-teachers-pervasive-in-many-schools-2/.

100 Anian Zhang, Lauren Musu-Gillette, and Barbara A. Oudekerk, "Indicators of School Crime and Safety: 2015" (May 2016)// http://nces.ed.gov/pubs2016/2016079.pdf.
} 
results in an inadequate representation of safety issues, which in turn, makes it more difficult to formulate effective solutions by adopting policies or school regulations. ${ }^{101}$

\subsubsection{BEYOND THE GENERAL PERCEPTION OF THE NEGATIVE EFFECT}

Legal definitions of bullying extend well beyond its negative effect on an individual victim and his or her property. Many states explicitly refer to the negative effect of bullying in the context of 'material and substantial disruption of the education process,' or 'disorderly operation of a school,' or creating 'hostile,' 'intimidating,' or 'threatening environment in the school,' 'interfering with the educational performance, opportunities or benefits. ${ }^{102}$ For example, the definition of bullying in the state legislation of Arkansas even refers to 'substantial interference [...] with a public employee's role in education.' ${ }^{103}$ Legislation of Oklahoma in this respect protects the 'educational mission' of the school and 'the education of any student ${ }^{\prime 104}$ (not only the victim itself). Such notions imply that acts of bullying committed against one victim may have a detrimental effect on all members of the school community, both other students (not necessarily direct victims of an act of bullying) and the staff. And the legal approach toward a definition of bullying recognizes that such the acts interfere with emotional and educational integrity both directly and indirectly.

It is relevant to point out that in some instances the legislator even defines (and it is done within the same statutory definition of bullying) what is meant by 'substantial disruption' of the orderly operation of the school. For example, cessation of instruction of educational activities, inability to focus on learning (both by students and the staff), to function as an educational unit because of hostile environment, need of severe repetitive measures ${ }^{105}$ are all notions regarded as substantial interference with the orderly operation of the school as a unit. In such instances the legal definition of bullying takes into consideration the entire process of education and the general well-being of the school environment.

\footnotetext{
101 Tim Walker, "Violence Against Teachers - An Overlooked Crisis?" (February 2013) // http://neatoday.org/2013/02/19/violence-against-teachers-an-overlooked-crisis-2/.

102 Such notions are explicitly cited in the legislations of all the states, with exception of Colorado, Indiana, Missouri, Nebraska, Nevada, Utah, Vermont, Virginia, see Table 1 for relevant states and citation references.

103 Arkansas House Bill, supra note 41.

104 Oklahoma House Bill, supra note 72.

105 Arkansas House Bill, supra note 41.
} 


\subsubsection{THE BROADER PERCEPTION OF BULLYING AT SCHOOL IN REGARD TO ITS PLACE (TERRITORY) AND TIME OF INCIDENCE}

Another important aspect of the legal definition of bullying is its reference to the time and location of when and where the act was committed and whether it shall be regarded as an act of bullying at school. Logically, the notion of bullying at school indicates that an act is committed at an actual school, i.e., school building and premises, playgrounds, and whatever is generally perceived as the school property used during the process of education. Legal definitions of bullying, however, point out certain aspects in regard to location, that are not generally perceived as school property per se used for the purposes of education. Large number of states' legislation not only refer to the general notion of 'school property,' but also to notions such as, for example, 'school busses,' 'school vehicles, [...] designated school bus stop,' ${ }^{106}$ 'school-provided transportation,'107 'places immediately adjacent to school grounds,',108 etc.

The definitions of some states acknowledge the importance of the time during which the act of bullying may occur. For example, it is generally perceived that a child is at school for the purposes of education during the regular school hours of a school day. This general perception is reflected in the legal definition of bullying. However, the legal definitions also refer to the broader concept of the process of compulsory education, i.e., in regard to bullying school time is referred to any time 'during any education program,' 109 'during the school day, [...] at a school sponsored activity, or before or after the school day, ${ }^{110}$ or 'school activities or sanctioned events,' 111 'school-sponsored functions'112 or ' related activities.' 113

Expanding the legal definition of bullying in regard to more explicit definitions of both time and place of its incidence, is material in understanding that the right to compulsory education is related directly not only to receiving education in form of knowledge and skill according to the state regulated education program at a school during a class on a school day, but also to a much broader scope of other rights such as accessing education, being safe not only during the official learning hours, but also outside of a class, after school hours, while interacting with other fellow students during school-related activities. Such explicit reference by state law in regard to the time and place of incidence of bullying strongly suggests that the law

\footnotetext{
106 Colorado Senate Bill, supra note 43.

107 Tennessee Senate Bill, supra note 77.

108 Oregon House Bill, supra note 73.

109 Florida Senate Bill, supra note 46.

110 Vermont House Bill, supra note 80.

111 Colorado Senate Bill, supra note 43.

112 Alabama House Bill, supra note 39.

113 Georgia House Bill, supra note 47.
} 
in education carries out a function of advocating for the rights of the learners during the whole process of acquiring compulsory education.

\subsubsection{OTHER RELEVANT CHARACTERISTICS OF BULLYING AT SCHOOL}

Some definitions of bullying suggest that a student who personally violates statutory provisions in regard to bullying may be guilty of an infraction, ${ }^{114}$ thus suggesting, though not specifying, that such violation may be a punishable one, and raising the question of responsibility of the student in breach of the statutory provisions. Many states define acts of 'harassment,' 'intimidation' and 'bullying' under one legal definition. ${ }^{115}$ Retaliation against a student or an employee asserting or alleging and act of bullying is in some instances also explicitly described as bullying. ${ }^{116}$

Some definitions of bullying refer to its discriminative character. For instance, several definitions explicitly define bullying as an act motivated by any ${ }^{117}$ actual, perceived, ${ }^{118}$ differentiated, ${ }^{119}$ or distinguishing ${ }^{120}$ personal characteristic of the victim, without limitation or including race, national origin, marital status, sex, sexual orientation gender identity, religion, ancestry, physical attribute, socioeconomic status, familial status, or physical or mental ability or disability, ${ }^{121}$ as well as pupil's behaviors or beliefs, or association with a person and based on this other person's characteristics, behaviors, or beliefs. ${ }^{122}$ One jurisdiction explicitly states that the act 'need not to be based' on any form of discrimination in order for it to be qualified as an act of bullying. ${ }^{123}$

\section{CONCLUSIONS}

Analysis of the state laws that have been adopted with the purpose to prevent bullying shows that it is feasible and productive for a legislator to provide a more or

\footnotetext{
114 Idaho House Bill, supra note 49.

115 There are nineteen states that define bullying, harassment and intimidation under the same definition: (1) Alabama House Bill, supra note 39; (2) Alaska House Bill, supra note 40; (3) Idaho House Bill, supra note 49; (4) Iowa Senate File, supra note 52; (5) Louisiana Laws Revised Statue, supra note 55; (6) Maryland House Bill, supra note 57; (7) Mississippi Senate Bill, supra note 61; (8) New Jersey Assembly Bill, supra note 67; (9) New York Assembly Bill No. 3661, Dignity for All Students Act1987B, N.Y. Educ. Law §11 (2011); (10) North Carolina Senate Bill, supra note 69; (11) Ohio House Bill, supra note 71; (12) Oklahoma House Bill, supra note 72; (13) Oregon House Bill, supra note 73; (14) Rhode Island House/Assembly Bill No. 7213, R.I. Gen. Law §16-21-26(2008); (15) South Carolina House Bill, supra note 75; (16) Tennessee Senate Bill, supra note 77; (17) Washington Substitute House Bill, supra note 82; (18) West Virginia House Bill, supra note 83; (19) Wyoming House Bill, supra note 84.

116 Florida Senate Bill, supra note 46; Illinois Senate Bill, supra note 50.

117 Alabama House Bill, supra note 39.

118 Arkansas House, supra note 41.

119 Mississippi Senate Bill, supra note 61.

120 New Jersey Assembly Bill, supra note 67.

121 Maryland House Bill, supra note 57.

122 New Hampshire Senate Bill, supra note 66.

123 New Mexico Department of Public Education, supra note 68.
} 
less detailed legal definition of bullying at school, which can include different aspects of bullying in regard to the risks associated with such behavior.

The legal definition of bullying deviates from the two generally accepted component parts of definition of bullying - repetitiveness and imbalance of power. It can concluded that the legal definition does not have to (and in vast majority of the state jurisdictions does not) refer to all three component parts (negativity, repetitiveness and imbalance of power) of the universally recognized and applied definition penned by Dan Olweus.

The divergence of the legal definition of bullying at school from the generally accepted definition brings out its distinctive all-encompassing character. The legal definitions, as discussed above, encompass major aspects of bullying at school, taking into consideration both the victim, the bystanders, the student-body, the staff of the school, location, etc. And while bullying itself is a 'non-criminal' activity, ${ }^{124}$ the emergence of regulatory legislation with specific regard to bullying as a form of negative behavior at school shows the importance of the legal imperatives. ${ }^{125}$ The way these legal definitions of bullying and other related terms are drafted, they by and large raise the status of a student (or all students affected) by protecting and advocating for his or her rights, as well as the rights of the members of the school community in general.

The emerging forms of bullying at school rightly suggest that the component part of the definition of bullying referring to the continuous character of bullying over time should be revisited and include not only the long-standing ${ }^{126}$ pattern of negative behavior towards the victim, but any incidence of an act of bullying. This observation is pertinent for better comprehension on the part of the learners and other members of the school community as to what bullying is. As a rule, schools on individual levels as well as policy makers tend to rely largely on the generally accepted definition which requires that action towards the victim to be 'repetitive over time' (based on the concept developed by Olweus). Therefore, providers of education that have adopted measures (in forms of policies, rules, education) should consider revising the definition of bullying accordingly.

\section{BIBLIOGRAPHY}

1. Bagdonas, Albinas, and Indrè Padarauskaitè. "Paaugliu kūno vaizdas ir patyčiu patirtis" (Body-Image and Bullying in Adolescents). Psichologija 46 (2012): 45-59.

124 Virginia Board of Education, "Student Conduct Policy Guidelines" (June 2009) // http://www.doe.virginia.gov/boe/meetings/2009/07_jul/agenda_items/item_p.pdf.

125 Neville Harris, supra note 1: 34 .

126 Stan Davis and Julia Davis, supra note 9, 9. 
2. Batche, George M., and Howard M. Knoff. "Bullies and Their Victims: Understanding A Pervasive Problem in the Schools." School Psychology Review 23 (2004): 165-175.

3. Chester, Kayleigh L., Mary Callaghan, Alina Cosma, Peter Donnelly, Wendy Craig, Sophie Walsh, and Michal Molcho. "Cross-national time trends in bullying victimization in 33 countries among children aged 11, 13 and 15 from 2002 to 2010." European Journal of Public Health Vol. 25 (Supplement 2, 2015): 61-64//

DOI: doi:10.1093/eurpub/ckv029.

4. Davis, Stan, and Julia Davis. Schools Where Everyone Belongs: Practical Strategies for Reducing Bullying. $2^{\text {nd }}$ ed. Champaign, IL: Research Press, 2007.

5. Girdvainis, Giedrius, and Rasa Pocevičienè. "Patyčiu problema ir jos prevencijos bendrojo lavinimo mokykloje analizė" (Analysis of Problem of Bullying and its Prevention at Comprehensive School). Socialiniai mokslai. Jaunuju mokslininku darbai 4(25) (2009): 127-135.

6. Girdvainis, Saulius. "Patyčios tarp mokiniu bendrojo lavinimo mokykloje: samprata, dalyviai, priežastys ir padariniai" //

http://www.su.lt/bylos/mokslo_leidiniai/jmd/2013_2_40/girdvainis.pdf.

7. Harris, Neville. "Pupil Bullying, Mental Health and the Law in England": 31-58. In: Neville Harris and Paul Meredith, eds. Children, Education and Health, International Perspectives on Law and Policy. ASHGATE, 2005.

8. Hoover, John H., Ronald L. Oliver, and Keith A. Thomson. "Perceived victimization by school bullies: New research and future directions." Journal of Humanistic Education and Development 32 (1993): 76-84.

9. Jonynienè, Živilè Vilma, Agnè Bartkutè and Tomas Butvilas. "Teisinis švietimas kaip prevencinè priemonè prieš patyčias mokyklose: mokiniu ir mokytoju sampratos" (Legal Education as the Prevention for Bullying at Schools: Conceptions of Students and Teachers). Socialinis darbas/Social Work 10(2) (2011): 217-228.

10. "Key Components in State Anti-Bullying Laws." stopbullying.gov (March 2014) //

http://www.stopbullying.gov/laws/key-components/index.html.

11. Kulbarsh, Pamela. "Bullycide: Suicide as a Result of Bullying" (January, 2012) // http://www.officer.com/article/10611621/bullycide-suicide-as-a-result-ofbullying.

12. Long, Cindy. "Bullying of Teachers Pervasive in Many Schools" (May 2012) // 
http://neatoday.org/2012/05/16/bullying-of-teachers-pervasive-in-manyschools-2/.

13. Marr, Neil, and Tim Field. Bullycide: Death at Playtime - An Expose' of Child Suicide Caused by Bullying. Success Unlimited, 2001.

14. Olweus, Dan. "Bully/Victim Problems in School: Facts and Intervention." European Journal of Psychology of Education Vol. XII (1997): 495-510.

15. Olweus, Dan. "Bullying - Victim Problems Among School Children: Basic Facts and Effects of a School Based Intervention Programs": 411-448. In: Debra J. Pepler and Kenneth H. Rubin, eds. Development and Treatment of Childhood Aggression. Hillsdale, NJ: Lawrence Elbraum Associates, Inc., 1991.

16. Olweus, Dan. Bullying at School: What We Know and What Can We Do. Oxford, England: Blackwell Publishers, 1993.

17. Oxford University Press. "Definition of persistent in English" (2016) // http://www.oxforddictionaries.com/us/definition/american_english/persistent.

18. Parkes, Aisling. Children and International Human Rights Law, The Right of the Child to be Heard. Routledge, 2013.

19. "Policies \& Laws." stopbullying.gov (May 2015) // http://www.stopbullying.gov/laws/index.html.

20. Povilaitis, Robertas, and Jurgita Valiukevičiūtè. Patyčiu prevencija mokyklose (Prevention of Bullying at Schools). Edited by Laima Bulotaitè. Vilnius: Multiplex, 2006.

21. Pūras, Dainius. "Foreword": 5. In: Robertas Povilaitis and Jurgita Valiukevičiūtè. Patyčiu prevencija mokyklose (Prevention of Bullying at Schools). Edited by Laima Bulotaitè. Vilnius: Multiplex, 2006.

22. Targamadzè, Vilija, and Džiuginta Valeckienè. "Patyčiu bendrojo lavinimo mokyklose samprata: priežasčiu, formu ir pasekmiu diskursas" (Conception of Bullying at Comprehensive School: Discourse on Reasons, Forms and Consequences). Acta Pedagogica Vilnensia 19 (2007): 159-171.

23. The Stationery Office Limited. "School Standards and Framework Act 1998" (July 1998) //

http://www.legislation.gov.uk/ukpga/1998/31/pdfs/ukpga_19980031_en.pdf.

24. Timm, Paul. School Security, How to Build and Strengthen a School Safety Program. Edited by Brian Romer. Elsevier, 2015.

25. Turner, Heather A., David Finkelhor, Anne Shattuck, Sherry Hamby, and Kimberly Mitchell. "Beyond Bullying: Aggravating Elements of Peer Victimization Episodes." School Psychology Quarterly Vol. 30(3) (September 2015): 366-384 // http://dx.doi.org/10.1037/spq0000058. 
26. US Department of Education. "Key Policy Letters from the Education Secretary and Deputy Secretary" (December 2010) // http://www2.ed.gov/policy/gen/guid/secletter/101215.html.

27. USLegal, Inc. "Bullying Law \& Legal Definition" (2001-2016) // http://definitions.uslegal.com/b/bullying/.

28. Virginia Board of Education. "Student Conduct Policy Guidelines" (June 2009) //

http://www.doe.virginia.gov/boe/meetings/2009/07_jul/agenda_items/item_ p.pdf.

29. Walker, Tim. "Violence Against Teachers - An Overlooked Crisis?" (February 2013) //

http://neatoday.org/2013/02/19/violence-against-teachers-an-overlookedcrisis-2/.

30. Watkins, Chris, and Patsy Wagner. Improving School Behavior. London: Paul Chapman, 2000.

31. Whitted, Kathryn S., and David R. Dupper. "Best Practices for Preventing or Reducing Bullying in Schools." Children \& Schools 27 (3) (2005): 167-175.

32. Zaborskis, Apolinaras, et al. for WHO/HBSC Forum. "Lithuania: Youth Mental Health - From Research to Policies, Practice and Partnerships" (2007) // http://www.euro.who.int/_data/assets/pdf_file/0006/74769/Hbsc_Forum_20 07_Lithuania.pdf.

33. Zaborskis, Apolinaras, Lina Cirtautienè, and Nida Žemaitienè. "Bullying in Lithuanian schools in 1994-2002." Medicina (Kaunas) 41(7) (2005): 614-620.

34. Zhang, Anian, Lauren Musu-Gillette, and Barbara A. Oudekerk. "Indicators of School Crime and Safety: 2015" (May 2016) // http://nces.ed.gov/pubs2016/2016079.pdf.

\section{LEGAL REFERENCES}

1. Alabama House Bill No. 216, The Alabama Student Harassment Prevention Act No. 2009-571. Alabama Code §16-28B-3 (2009).

2. Alaska House Bill No.482, Harassment, Intimidation, and Bullying Policy. Alaska Statute $§ 14.33 .250$ (2006).

3. Arkansas House Bill No. 1708, Act to Define Bullying and Cyberbullying. Arkansas Code Annotated §6-18-514 (2005).

4. California Assembly Bill No. 606. California Education Code $\S 48900.1$ (2008).

5. Colorado Senate Bill No. 01-080, Concerning the Prevention of Bullying. Colorado Revised Statutes §22-32-109.1 (1) (b) (2001). 
6. Connecticut House Bill No. 5563. Connecticut General Statutes §10-222d (2006).

7. Delaware House Bill No. 7. Delaware Code Annotated Title 14 §4112D (2007).

8. Education and Inspections Act (2006) // http://www.legislation.gov.uk/ukpga/2006/40/pdfs/ukpga_20060040_en.pdf.

9. Florida Senate Bill No. 114. Florida Statutes Annotated §1006.147 (2007).

10. Georgia House Bill No. 84. Georgia Code Annotated §20-2-751.4 (1999).

11. Hawaii Administrative Rules. Department of Education §8-19-2.

12. Idaho House Bill No. 750aa. Jared's Law, Idaho Code §18-917a (2006).

13. Illinois Senate Bill No. 1026. 105 Illinois Compiled Statutes §5/27-23.7 (2006).

14. Indiana Senate Enrolled Act. Indiana Code Annotated §20-33-8-0.2 (2005).

15. Iowa Senate File No. 61. Iowa Code $\$ 280.28$ (2007).

16. Kansas House Bill No. 2310. Kansas Statutes Annotated §72-8256 (2008).

17. Kentucky House Bill No. 91, Chapter No. 25, The Golden Rule Act. Kentucky Revised Statues Annotated §158.148 (2008).

18. Louisiana Laws Revised Statute. Louisiana Revised Statutes Annotated $\S 17: 416.13$ (2010).

19. Maine Revised Statute Annotated. Title 20-A §6554 (2011).

20. Maryland House Bill No. 199. Maryland Education Code Annotated §7-424.3 (2008).

21. Michigan House Bill No. 241. Michigan Compiled Laws §380.1310b (2011).

22. Minnesota House File No. 826. Minnesota Statute §121A.031 (2014).

23. Mississippi Senate Bill No. 2015, Bullying and Harassing Behavior in Public Schools. Mississippi Code Annotated §37-11-67 (2010).

24. Missouri Senate Bill No. 894. Missouri Revised Statutes $§ 160.775$ (2006).

25. Montana Code Annotated. L. 2015, En. Sec. 2, Ch. 253, §20-5-208 (2015).

26. Nebraska Legislative Bill No. 205. Nebraska Revised Statutes §79-2,137 (2008).

27. Nevada Assembly Bill. Nevada Revised Statutes Annotated $\S 388.122$ (2009).

28. New Hampshire Senate Bill No. 360. New Hampshire Revised Statutes Annotated §193-F:3 (2000).

29. New Jersey Assembly Bill No. 3466, Chapter No. 122. N.J. Stat. Ann. §18A37-14 (2011).

30. New Mexico Department of Public Education Rule Title 6, Chap. 12, Part 7, Bullying Prevention. New Mexico Administrative Code §6.12.7.7 (2006).

31. New York Assembly Bill No. 3661, Dignity for All Students Act1987B. N.Y. Educ. Law $\S 11$ (2011). 
32. North Carolina Senate Bill No. 526, School Violence Prevention Act. N.C. Gen. Stat. §115C-407.5 (2006).

33. North Dakota House Bill No. 1465. North Dakota Century Code Chapter §15.119-17 (2011).

34. Ohio House Bill No. 276, Harassment, Intimidation, and Bullying Policy, Adopt Own. Ohio Rev. Stat. Ann. §3313.666 (2006).

35. Oklahoma House Bill No. 2215, School Bullying Prevention Act. Okla. Stat. Ann. Tit. 70, §24-100.3 (2002).

36. Oregon House Bill No. 2599. Or. Rev. Stat. §339.351(2009).

37. Pennsylvania House Bill No. 1067. 24 Pennsylvania Consolidated Statute §131303.1-A (2008).

38. Rhode Island House/Assembly Bill No. 7213. Rhode Island General Laws §1621-34 (2008).

39. Senate Bill No. 2404, Chapter 92, Bullying in Schools Act. Massachusetts General Laws Chapter §71, Section 370 (2010).

40. South Carolina House Bill No. 3573, Safe School Climate Act. South Carolina Code Annotated §59-63-120 (2006).

41. South Dakota Codified Law. §13-32-15 (2012).

42. Tennessee Senate Bill No. 283. Tenn. Code Ann. §49-6-4502 (2009).

43. Texas House Bill No. 1942. Texas Education Code Annotated $\S 37.0832$ (2011).

44. The Education (Independent School Standards) Regulations (2010) // http://www.legislation.gov.uk/uksi/2010/1997/pdfs/uksi_20101997_en.pdf.

45. Utah House Bill No. 325. Utah Code Annotated §53A-11a-102 (2008).

46. Vermont House Bill No. H629. Vermont Statute Annotated Title 16, $\S 11(a)(32)(2004)$.

47. Virginia Code Annotated. § 22.1-276.01 (2013).

48. Washington Substitute House Bill No. 2801, Harassment, Intimidation, and Bullying. Wash. Rev. Code §28A.300.285 (2010).

49. West Virginia House Bill No. 3225. West Virginia Code Annotated §18-2C-2 (2011).

50. Wyoming House Bill No. 0223. Wyo. Stat. Ann. §21-4-312 (2009). 